Check for updates

Cite this: RSC Adv., 2019, 9, 15582

Received 30th November 2018 Accepted 10th April 2019

DOI: $10.1039 / c 8 r a 09849 b$

rsc.li/rsc-advances

\section{Enhancement of rotator cuff tendon-bone healing using combined aligned electrospun fibrous membranes and kartogenin}

\author{
Qi Zhu, † $^{a}$ Zhijie Ma, $\dagger^{\text {bc }}$ Haiyan Li, (D) bc Haiming Wang*a and Yaohua He (iD *a
}

Rotator cuff tear (RCT) is a major challenging shoulder disease because the fibrocartilage zone is hard to regenerate in the enthesis. Electrospun membranes with aligned nanofibers can guide the ordered tissue regeneration and kartogenin (KGN) is able to stimulate chondrocyte differentiation of mesenchymal stem cells. In this study, we fabricated a functional engineered scaffold for regenerating tendon-bone enthesis in RCTs by taking advantage of both the structural guiding ability of aligned nanofibers and the biology effects of KGN. Polycaprolactone (PCL) fibrous membranes with aligned nanofibers loaded with or without KGN were fabricated using electrospinning and characterized using scanning electron microscopy (SEM). The release of KGN from PCL membranes and the effects of KGN on differentiation of mesenchymal stem cells were investigated. Results indicated that $100 \mu \mathrm{M} \mathrm{KGN-loaded} \mathrm{PCL} \mathrm{(KGN-PCL)} \mathrm{membranes} \mathrm{significantly}$ stimulated chondrogenic and tenogenic differentiation of rat bone marrow stromal cells. In addition, after PCL and $100 \mu \mathrm{M}$ KGN-PCL membranes were applied to an acute rat RCT model, KGN-PCL membranes promoted fibrocartilage formation and collagen organization as well as increased cross-sectional area and load failure. In conclusion, PCL electrospun fibrous membranes with aligned nanofibers and KGN could be an effective tissue engineering scaffold to enhance tendon-bone healing in RCTs.

\section{Introduction}

Rotator cuff tear (RCT) is the most common shoulder disease that causes reduced shoulder strength and function-limiting pain. Despite the advancement of surgical treatments, the failure rates of rotator cuff repairs are still concerning to surgeons. ${ }^{1-6}$ Previous clinical studies have demonstrated that tendon-bone healing is difficult to achieve. Tendon-bone junctions (TBJs) are unique structures composed of tendon, encalcified fibrocartilage, calcified fibrocartilage, and bone. ${ }^{7}$ Previous studies have shown that natural healing and surgical treatment are insufficient for fibrocartilage regeneration. ${ }^{8-10}$ According to the results of histopathological studies, the fibrocartilage zone cannot completely regenerate when the TBJs are healing, resulting in weak mechanical strength and possible re-injury in the repaired interface. ${ }^{8,9}$ Therefore, recent studies have focused on strategies to restore the fibrocartilage

\footnotetext{
${ }^{a}$ Department of Orthopedics, Shanghai Jiao Tong University Affiliated Sixth People's Hospital, 600 Yishan Road, Shanghai 200233, China. E-mail: heyaohua@sjtu.edu. cn; drwanghaiming@126.com

${ }^{b}$ Shanghai Jiao Tong University Affiliated Sixth People's Hospital, School of Biomedical Engineering, Shanghai Jiao Tong University, 1954 Huashan Road, Shanghai 200030, China

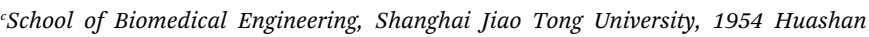
Road, Shanghai 200030, China

$\dagger$ The two authors contributed to the work equally.
}

transition zone of the TBJs after rotator cuff tear, which is already attracting increasing attention. ${ }^{11,12}$

Recently, a small molecule drug named kartogenin (KGN) was found to promote chondrocyte differentiation of primary mouse mesenchymal stem cells (MSCs). ${ }^{13}$ KGN has been reported to induce MSCs to express a set of chondrogenic genes, which indicates that KGN could induce differentiation of MSCs to chondrocytes. ${ }^{14}$ As a result of the chondrogenesis induction properties, KGN has been found to be a new treatment of cartilage defects. ${ }^{15}$ In addition, a recent study showed that KGN enhanced healing of the TBJs in experimental rat achilles when it was injected into the experimental area. ${ }^{14} \mathrm{KGN}$ treatment promoted fibrocartilage formation at the tendon-bone interface to regenerate the fibrocartilage zone of the native enthesis and increased the biomechanical properties of tendon-bone enthesis. ${ }^{\mathbf{1 6}}$ However, single topical injection of KGN in wound areas may not be an optimal treatment route strategy. The KGN solution may be washed away from wound sites since the body fluid system is dynamic and circulation occurs. Therefore, cartilage tissue in the tendon-bone enthesis would not be formed because of low KGN concentration. In addition, repeated injection may cause injury to patients. To maintain effective concentrations of KGN at wound sites, a sustained delivery system is necessary. ${ }^{17}$

Biological scaffolds have been widely used in tissue engineering and drug delivery. ${ }^{\mathbf{1 8 - 2 4}}$ Electrospun scaffolds represent a promising substrate in tissue engineering and drug delivery 
because of their high porosity and large surface ratios. ${ }^{25,26}$ Furthermore, compared with electrospun fibrous scaffolds containing random nanofibers, electrospun fibrous scaffolds containing aligned nanofibers have been reported to enhance tenogenesis in tendon-bone regeneration. ${ }^{27-30}$ Specifically, the aligned electrospun nanofiber structure provides a constructive microenvironment to promote tenogenesis and created mechanical anisotropy that similar to tendon mechanical properties. ${ }^{29,30}$

Considering the advantages of electrospun fibrous membranes with aligned nanofibers for RCT repair and drug delivery, in this study, we designed electrospun fibrous membranes with aligned nanofibers, which were then used as a locally controlled release system for KGN. PCL has been widely used for biomedical applications because of its slow degradability, good biocompatibility, and superior rheological and viscoelastic properties. ${ }^{31}$ Besides, PCL has been reported to be able to support cell attachment including mesenchymal stem cells and chondrocytes. ${ }^{32}$ In addition, PCL has been used in cartilage repair because of its good mechanical properties and its slow degradability for long-term implantation into the injury site. ${ }^{33}$ Therefore, in the present study, PCL electrospun fibrous membranes with aligned nanofibers and an appropriate amount of KGN were fabricated (KGN-PCL). The release behavior of KGN from the KGN-PCL membranes was evaluated and the effects of KGN on chondrogenic and tenogenic differentiation of MSCs were investigated. Then, PCL membranes and KGN-PCL were implanted into an acute rat RCT model to evaluate the effects of different materials on the repair of RCT.

\section{Materials and methods}

\section{Fabrication of aligned electrospun fibrous membrane}

$9.62 \mathrm{mg}$ of KGN (Selleck, USA) was dissolved in $3 \mathrm{ml}$ dimethyl sulfoxide (Aladdin) to obtain a $10 \mathrm{mM}$ KGN stock solution. Besides, 0.3 g PCL (Dai gang Biomaterial Co., Ltd. Jinan, China) was dissolved in $3 \mathrm{ml}$ trichloromethane and stirred at room temperature to obtain PCL solution with concentration of $0.1 \mathrm{mg} \mathrm{ml}^{-1}$. Then, different volume of KGN stock solution was added into $3 \mathrm{ml}$ PCL solution and the mixed solution was stirred overnight in order to obtain KGN-PCL working solutions with KGN concentration of $10 \mu \mathrm{M}, 50 \mu \mathrm{M}$ and $100 \mu \mathrm{M}$, respectively. The KGN-PCL solution was then electrospun by an electrospinning machine (TEADFS-103 Xinrui Technology Co., Ltd Beijing). Briefly, the KGN-PCL working solution was added into a $5 \mathrm{ml}$ syringe connected with an injection pump. The solution was delivered to the needle and injected with an injection rate of $3 \mathrm{ml} \mathrm{h}^{-1}$ under a voltage was $15 \mathrm{kV}$. The aligned KGN-PCL electrospun fibers were collected using a rotating drum covered with aluminum foil and the rotary speed of the collecting drum was $2000 \mathrm{rpm}$. Aligned PCL electrospun fibers were fabricated with the same method.

\section{Wettability of aligned electrospun fibrous membrane}

Water contact angle (WCA) measurement of electrospun fibrous membranes was performed using sessile drop method by a video-enabled goniometer (DSA100, KRÜSS, Germany). At least $300 \mu \mathrm{l}$ distilled water was placed randomly at different locations of each sample. After settling the droplets on the fibrous membrane sheets with no noticeable changes in their shapes, the projected images of the droplets were analyzed for determining contact angle.

\section{Release behavior of KGN from aligned KGN-PCL electrospun fibrous membranes}

$20 \mathrm{mg}$ of as-prepared KGN-PCL membranes loaded with different amount of KGN was incubated in $2 \mathrm{ml}$ PBS buffer solution at $37^{\circ} \mathrm{C}$ in a shaking incubator. At $1 \mathrm{~d}, 2 \mathrm{~d}, 5 \mathrm{~d}, 10 \mathrm{~d}, 15$ $\mathrm{d}$ and $20 \mathrm{~d}, 300 \mu \mathrm{l}$ solution was collected and equal amount of fresh PBS was added. The OD value of KGN in the collected solution was measured by automatic microplate reader (Tecan infinite m200pro) at $278 \mathrm{~nm}$ and the cumulative released amount of KGN was calculated according to the standard curve. Each experiment was conducted for three times.

\section{Cell culture}

rBMSCs were isolated from femur bone according to the procedures reported in previous study, ${ }^{\mathbf{3 4 , 3 5}}$ which was approved by the Animal care Committee of Shanghai Jiao Tong University. rBMSCs were cultured in low-glucose DMEM (Hyclone, USA) supplemented with $10 \%$ fetal bovine serum (GIBCO, USA) and $1 \%$ antibiotics (penicillin $100 \mathrm{U} \mathrm{ml}^{-1}$, streptomycin $100 \mathrm{~g} \mathrm{ml}^{-1}$, GIBCO, USA) in a cell incubator with $5 \% \mathrm{CO}_{2}$ at $37^{\circ} \mathrm{C}$. The third to fifth passages of rBMSCs were used in this study.

\section{Cell proliferation assay}

Proliferation of rBMSCs on aligned PCL electrospun fibrous membranes with different amount of $\operatorname{KGN}(0,10,50$ and $100 \mu \mathrm{M})$ was measured by a cell viability assay kit (Cell Counting kit-8, CCK-8; Dojindo Technologies Japan). Briefly, aligned electrospun fibrous membranes containing different amount of KGN were settled in wells of a 96-well plate and rBMSCs at a density of $3 \times 10^{3}$ cells per well were cultured on the membranes in complete DMEM medium for 1, 4, 7 days, respectively. At each time point, the cell culture medium in each well was replaced with $100 \mu \mathrm{l}$ of culture medium containing 10\% CCK- 8 solution and the cells were continued to be cultured for $2 \mathrm{~h}$. For each condition, five wells were applied. The plate was then read by a spectrophotometric microplate reader (Thermo USA) to obtain absorbance value at $450 \mathrm{~nm}$ of the solution in each well. The obtained data (OD values) represents the metabolic activity of cells and reflects the number of living cells.

\section{Real-time quantitative reverse transcription PCR (qRT-PCR) analysis}

To evaluate the effects of different membranes on chondrogenic and tenogenic differentiation of rBMSCs, cells were cultured on aligned PCL electrospun fibrous membrane containing 0, 10, 50 and $100 \mu \mathrm{M}$ of KGN, respectively in 6-well plate for 2 weeks. The medium was changed every 3 days. The expression of chondrocyte-related genes (Aggrecan, collagen type II and SRY- 
box 9) and tenocyte-related gene (scleraxis bHLH transcription factor) was measured by qRT-PCR. Briefly, RNA was isolated from BMSCs using a RNeasy Mini Kit (Qiagen, Valencia, CA, USA) for synthesizing first-strand cDNA by reverse transcription. The incubation program for cDNA synthesis was as following: heating at $65{ }^{\circ} \mathrm{C}$ for $5 \mathrm{~min}$, then cooling at $4{ }^{\circ} \mathrm{C}$ for $1 \mathrm{~min}$, reverse transcription at $42{ }^{\circ} \mathrm{C}$ for $60 \mathrm{~min}$ and a final incubation at $80^{\circ} \mathrm{C}$ for $5 \mathrm{~min}$. The obtained cDNA was then used for qRT-PCR, which was performed on Applied Biosystems 7300 (Applied Systems, USA). The PCR cycling consisted of 40 cycles at $95{ }^{\circ} \mathrm{C}$ for $15 \mathrm{~s}$ and $60{ }^{\circ} \mathrm{C}$ for $1 \mathrm{~min}$. Glyceraldehyde 3-phosphate dehydrogenase (GAPDH) was used as internal reference. The relative gene expression levels were analyzed by the $2^{-\triangle \mathrm{DDC} C_{\mathrm{T}}}$ method. Each experiment was repeated three times to obtain a mean gene expression level.

\section{Animal studies}

One hundred and thirty-five male Sprague-Dawley rats which were weighed between 400-500 g (2 months, Shanghai SIPPR-BK Laboratory Animal Co., Ltd., Shanghai, China) were used in the present study. All animal procedures were performed in accordance with the Guidelines for Care and Use of Laboratory Animals of Shanghai Jiao Tong University and approved by the Animal Care and Experimental Committee of Sixth People's Hospital affiliated to Shanghai Jiao Tong University School of Medicine. The animals were randomly into three groups. The group $1(n=45)$ was considered as the control group in which the tendon was repaired to its anatomic footprint by transosseous repair. The group 2 and 3 (the experimental groups) not only underwent transosseous repair but also repaired with pure PCL membranes $(n=45)$ or KGN-PCL
$(100 \mu \mathrm{M})$ membranes. 15 animals per group were sacrificed at each time point $(2,4,8$ weeks) after the surgery and specimens were obtained for further analysis.

All the surgical procedures were performed according to the previous study. ${ }^{36,37}$ After the general anesthesia (intraperitoneal injection of Pentobarbital $40 \mathrm{mg} \mathrm{kg}^{-1}$ ), the rat was placed in the lateral decubitus position. A longitudinal incision on the anterolateral aspect of shoulder was made. Then, the deltoid muscle was separated and rotator cuff tendons were exposed. The supraspinatus tendon (superior of the scapula) was tied with a 40 Prolene suture (Ethicon, US) as a mark. The tendon was then sharply cut from the greater tuberosity. The footprint where the tendon was detached from was totally decorticated and debrided of the fibrocartilage remaining on the humeral head with bleeding. By using an 18-gauge needle, an anterior-posterior transverse tunnel from the proximal humeral to the footprint was created. The 3.0 Ethibond suture from end of the tendon was used to firmly tie over the footprint by passing through the tunnel, leading tendon attach its insertion site. The deltoid muscle and skin were closed finally. After the operation, all the animals received medication in next three days. In group 1, no further biomaterial was implanted. In group 2 and 3, the aligned PCL electrospun membranes and aligned KGN-PCL electrospun membranes were implanted in RCT defects, respectively.

\section{Histomorphometric analysis}

For histochemical analysis, totally forty-five animals (each time each group five animals) were sacrificed at 2, 4, 8 weeks postsurgery. The tendon-bone specimens were fixed at $10 \%$ neutral buffer formalin for 24 hours. After the decalcification treatment and
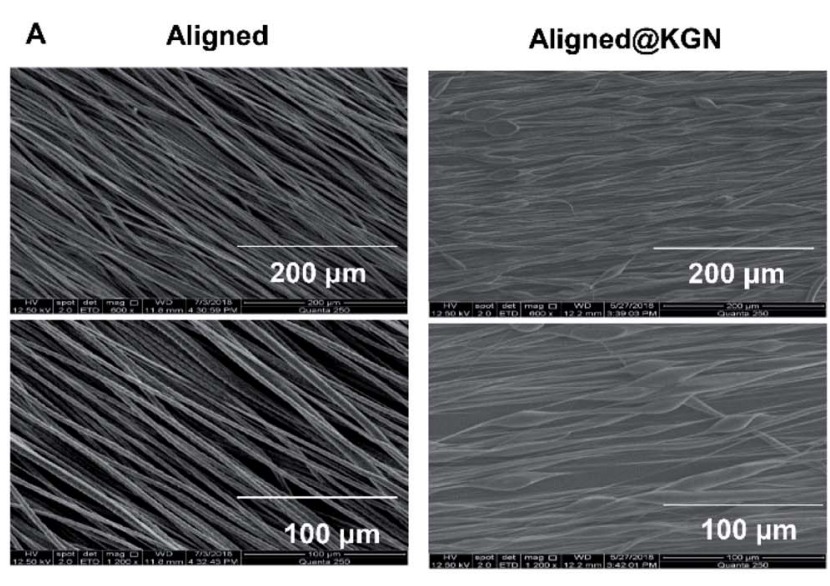

B

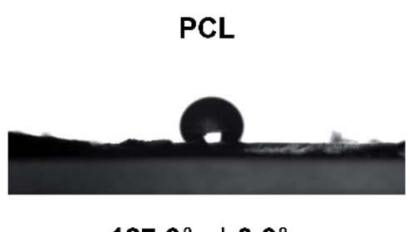

$127.6^{\circ} \pm 0.6^{\circ}$

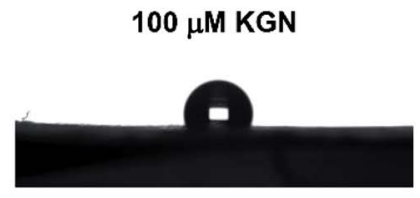

$110.8^{\circ} \pm 3.9^{\circ}$
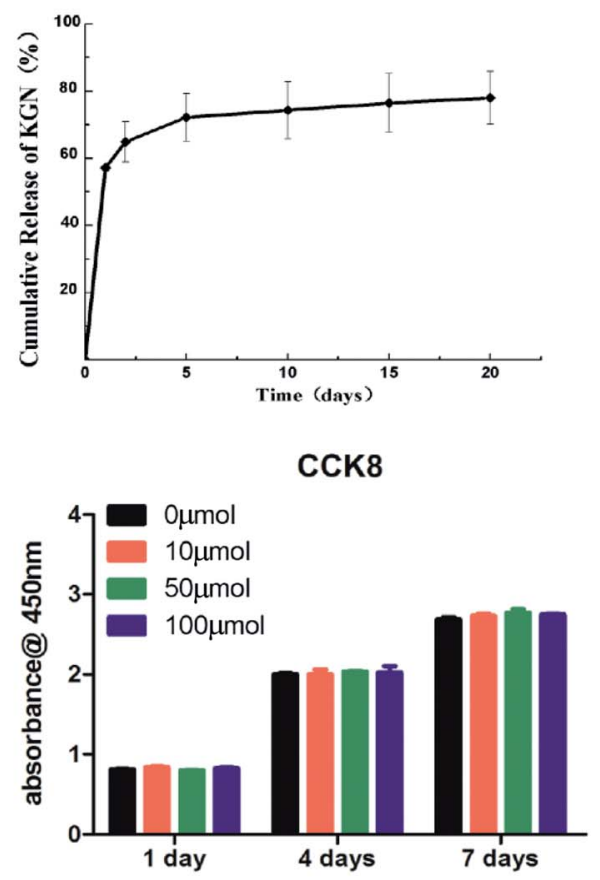

Fig. 1 (A) SEM image of aligned KGN-PCL scaffold and aligned PCL scaffold. (B) Wettability measurement of electrospun fibrous membranes in two groups. (C) In vitro release profile of KGN from aligned PCL scaffolds. (D) Proliferation of rBMSCs on different doses of aligned KGN electrospun fibrous membrane. There was no difference between $0 \mu \mathrm{m}$ and $10-100 \mu \mathrm{m}$ groups. 
paraffin embedded, the specimens were cut into $5 \mu \mathrm{m}$ thick sections in the coronal plane, including the supraspinatus tendon and tuberosity. Then, the specimens were processed for hematoxylin and eosin staining (HE), safranin O/fast green, and Masson trichrome staining. After staining, the specimens were observed by a microscope (Leica DM4000 B; Leica, Solms, Germany) and images were taken with a digital camera connected to the microscope.

In order to evaluate the new fibrocartilage formation, area of the tendon-bone interface was stained with safranin $\mathrm{O} / \mathrm{fast}$ green. Then, the metachromasia area was observed and photographed by the microscope. The area was measured with the Image Imaging software program (National Institutes of Health, Bethesda, MD) and analyzed to determine the novel fibrocartilage formation. Masson trichrome staining was used to observe the collagen fibril-formation in tendon-bone healing enthesis according to a previous research. ${ }^{38}$

For immunohistochemistry analysis, tissue specimens were cut into $5 \mu \mathrm{m}$ slices and fixed with cold acetone. After washing in PBS, the sections were incubated in with rabbit collagen I (COL, $1: 200$; Abcam) and rabbit collagen II together (COL2, 1 : 200; Abcam). Then sections were then rinsed in PBS for three times and incubated with secondary antibodies to detect the COL1 and COL2 in the slices for evaluating the new fibrocartilage formation in RCT repairs, all the staining sections was observed by the microscope and images were taken with a digital camera connected to the microscope.

\section{Biomechanical testing}

Forty-five animals were sacrificed for the biomechanical testing after surgery at 2, 4, 8 weeks. The humerus was cut from the soft tissues around the glenohumeral joint. The tendon of the supraspinatus muscle on the humeral head was completely retained. A digital caliper was used to measure the crosssectional area of supraspinatus tendon in the position of its insertion into the humerus head. The specimen was then tested by using a custom designed uniaxial testing system. The supraspinatus tendon and the humerus were firmly fixed on the testing machine with iron wire, keeping the tendon and humerus head in a straight line. The specimen was preloaded to $0.1 \mathrm{~N}$ at the beginning. Then, the specimen was loaded to failure at the rate of $0.14 \mu \mathrm{m} \mathrm{s}^{-1}$. We recorded the maximum load to failure and the failure site in order to reflect the healing degree of the supraspinatus tendon and the humerus head.

\section{Statistical analysis}

All the statistics were performed by SPSS software. One-way Anova was used to analyze the data, following by Fisher's

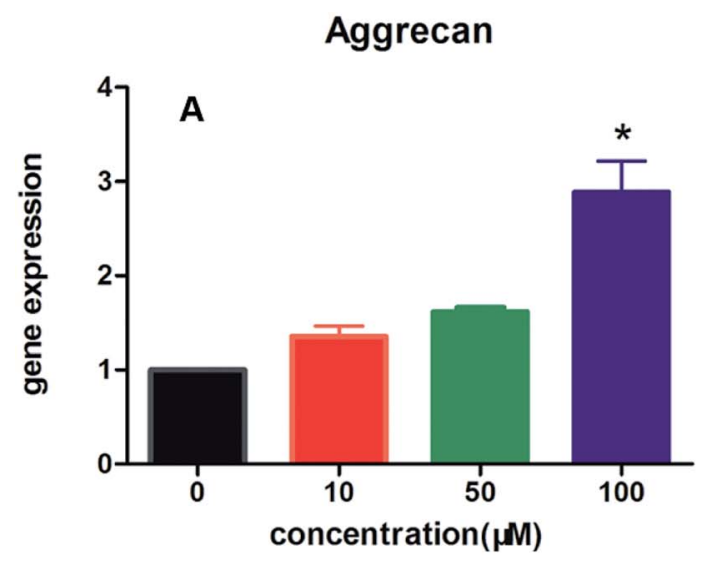

SOX-9

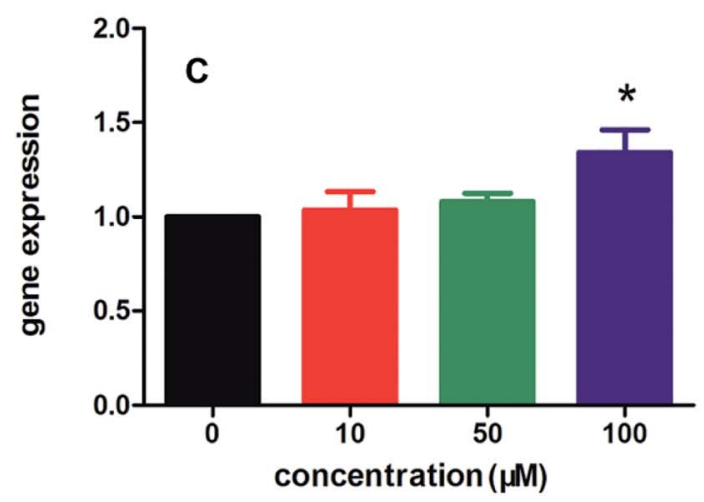

\section{COL2}

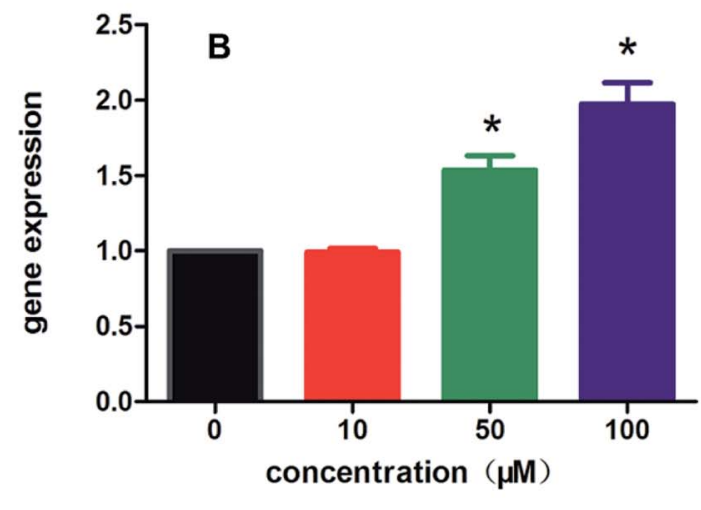

$\operatorname{scX}$

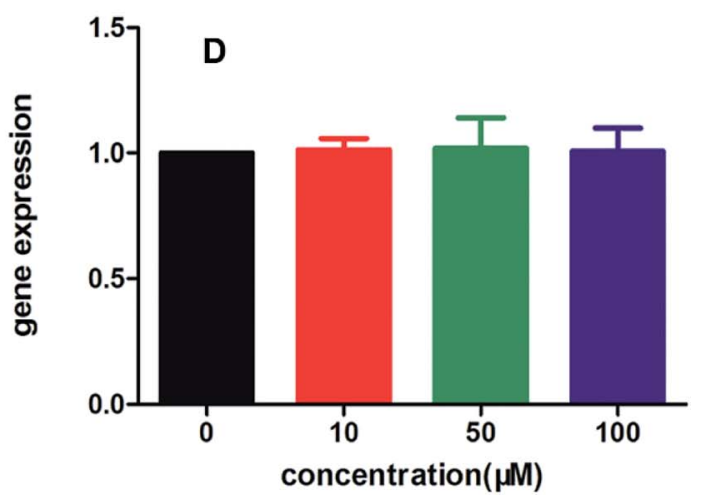

Fig. 2 Effect of different doses of KGN-PCL scaffold on the chondrocyte-related and tenocyte-related genes expression. (A-C) qRT-PCR analysis of Aggrecan, COL2, SOX9 in rBMSCs treated with KGN-PCL for 2 weeks. (*: significant differences between $0 \mu \mathrm{m}$ and $10-100 \mu \mathrm{m}$ groups $P<0.05)(D)$ qRT-PCR analysis of SCX in rBMSCs treated with different doses of KGN-PCL scaffolds. There was no difference between $0 \mu \mathrm{m}$ and $10-100 \mu \mathrm{m}$ groups. 
Table 1 Sequence of primers used for RT-PCR

\begin{tabular}{|c|c|c|}
\hline Gene & Forward primer & Reverse primer \\
\hline Aggrecan & 5'-CCTGGACAAGTGCTATGCTGG-3' & 3'-GCACCACTGACACACCTCGGAA-5' \\
\hline Sox9 & 5'-AGGGTTAAAGTGCCACAGAGGA-3' & 3'-AATGCTTTTCTGGTTCTTGGAGG-5' \\
\hline SCX & 5'-CAACGTGCTACTGGTGGGTGA-3 & 3'-GTCTTTCTGTCACGGTCTTTGCT-5' \\
\hline
\end{tabular}

least-significant difference (LSD) test for multiple comparisons. $p<0.05$ indicated that there was a significant difference between the two comparing group.

\section{Results}

\section{Characterization of electrospun fibrous membranes and KGN release behavior}

Aligned PCL electrospun fibrous membranes with or without KGN were fabricated using electrospinning technology. The morphology of as-obtained membranes was observed using scanning electron microscopy (SEM) and the results are showed in Fig. 1A. The two kinds of scaffolds maintained a porous structure with interconnected pores. However, nanofibers of the aligned KGN-PCL membranes had a uniform parallelism with a beaded structure, which indicated that KGN was encapsulated in the nanofibers. However, the nanofibers in the aligned PCL were smooth, bead free, and had a uniform parallelism.

Wettability measurement of electrospun fibrous membranes in two groups indicated that addition of kartogenin endowed electrospun fibrous membranes with better hydrophilicity and the results are showed in Fig. 1B. The water contact angle of the hybrid electrospun fibrous membranes with the addition of KGN is $110.8 \pm 3.9^{\circ}$, which is more hydrophilic as compared with that of pure PCL electrospun fibrous membranes (127 \pm $0.6^{\circ}$ ).

Fig. 1B shows the release behavior of KGN from aligned PCL electrospun membrane scaffolds. There was a burst release of KGN from aligned PCL scaffold as 57\% KGN was released after the KGN-PCL scaffolds were incubated in phosphate-buffered saline (PBS) for 24 hours. Over the following 20 days, KGN was constantly released and up to $80 \%$ was released by day 20 .
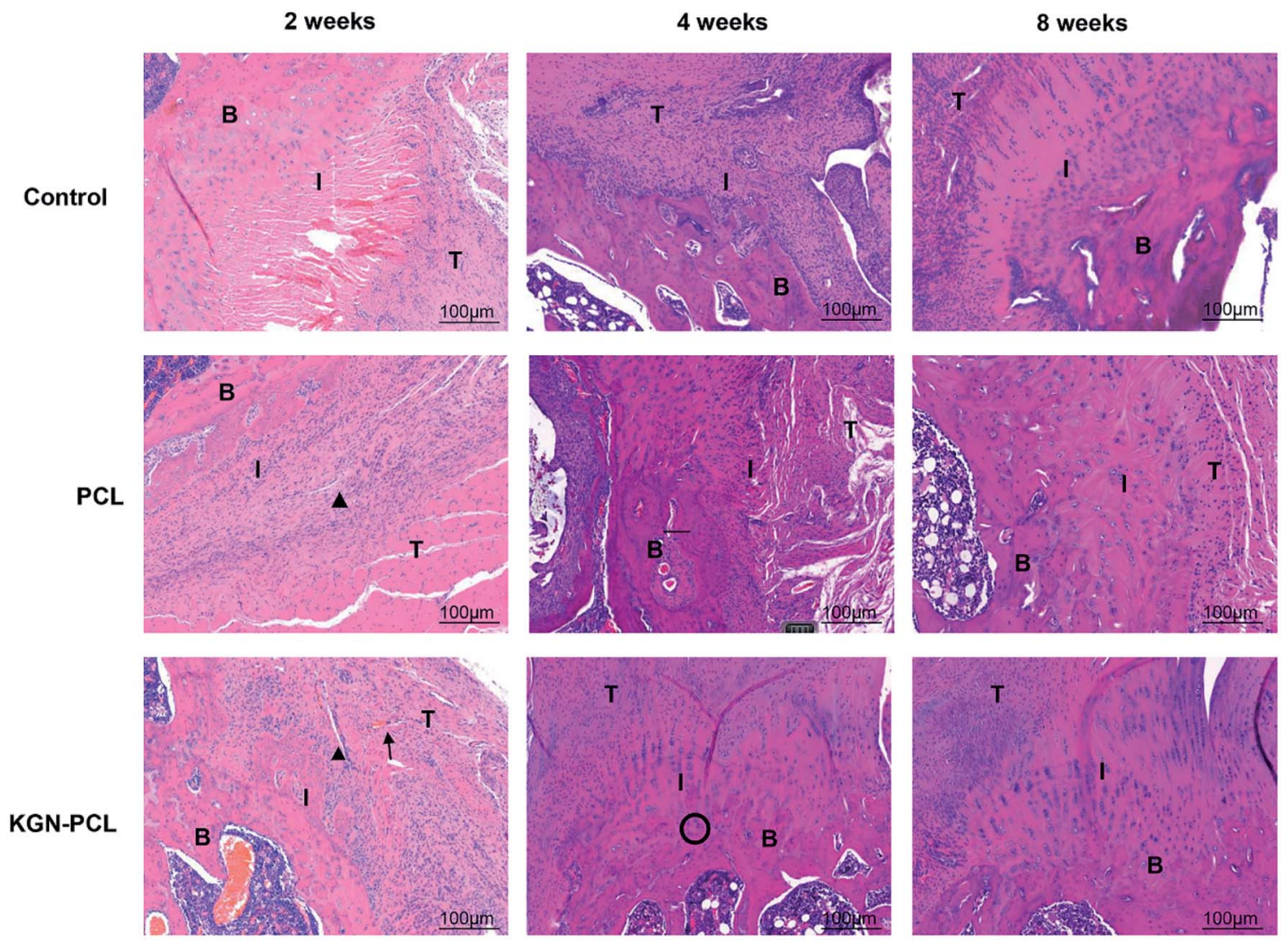

Fig. 3 Histology of rotator cuff tendon-bone healing enthesis, hematoxylin and eosin-stained at 2, 4, and 8 weeks after the surgery. T: tendon; I: interface; B: bone. Black triangle: degraded membrane fibrous; black arrow: new vascular; black cycle: chondrocytes. 
A

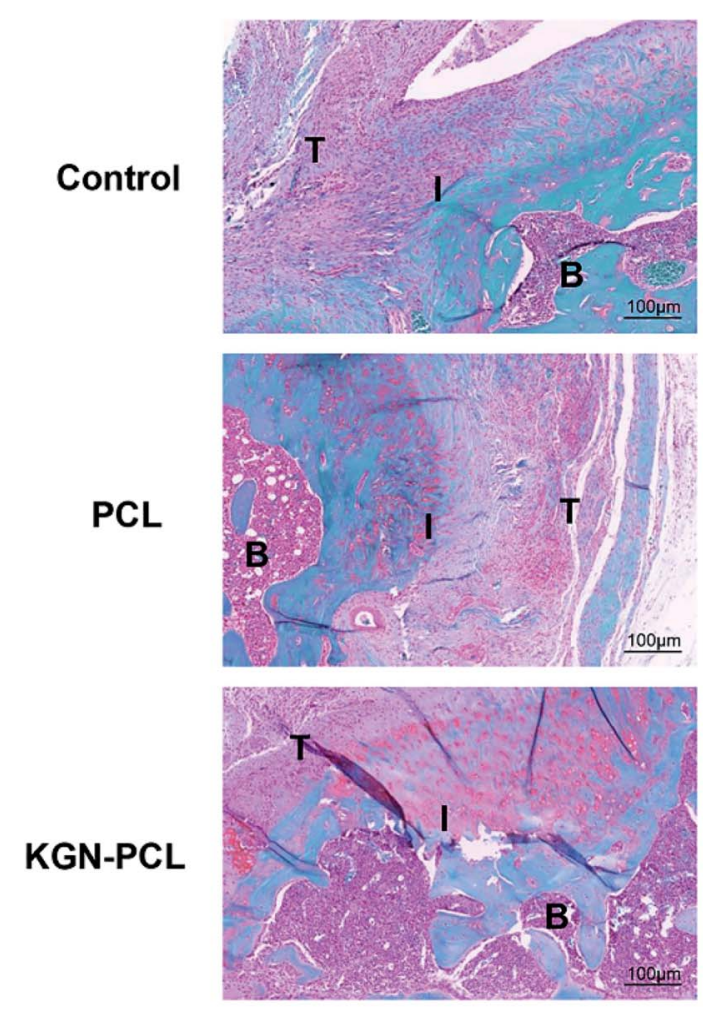

4 weeks
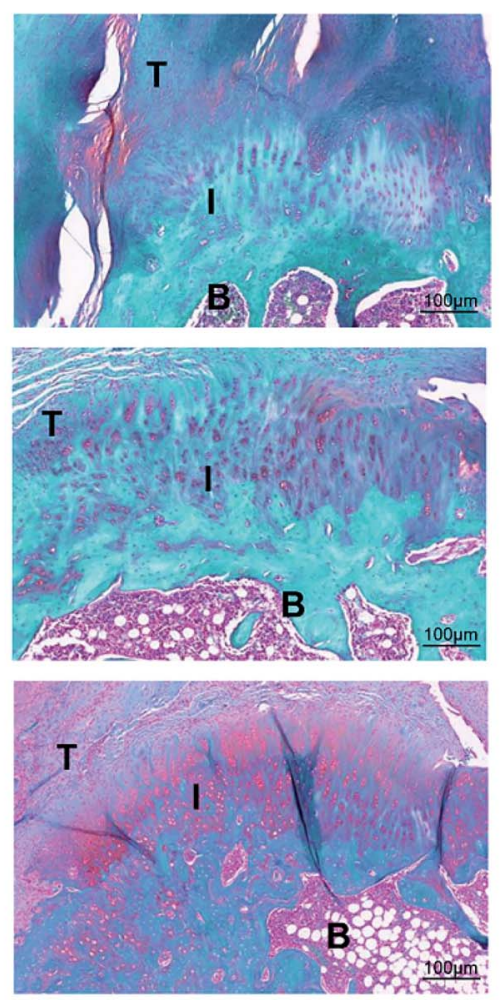

8 weeks
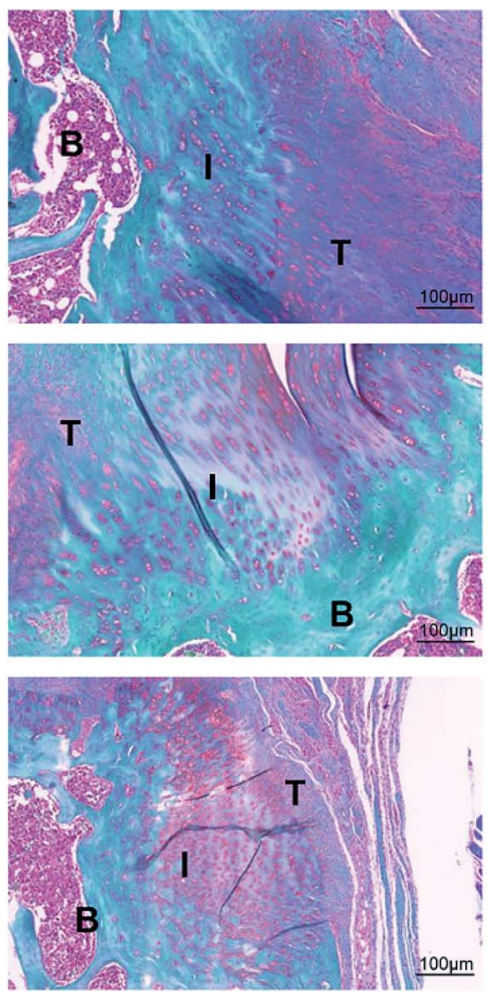

B

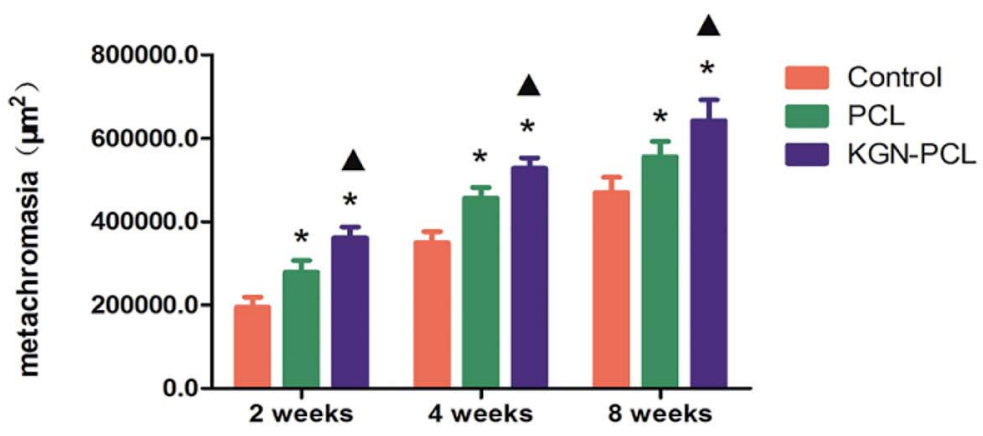

Fig. 4 Histology of rotator cuff tendon-bone healing enthesis, metachromasia with safranin $\mathrm{O}$-stained. (A) Representative area of tissue stained at the tendon-bone interface at 2, 4, and 8 weeks after the surgery. (B) Area of cartilage zone determined by metachromasia with safranin $O$ stained slides. The data are presented as the mean \pm standard deviation. T: tendon I: interface B: bone $(*: P<0.05$ versus control group; $\boldsymbol{\Lambda}$ : $P<$ 0.05 versus $\mathrm{PCL}$ group).

\section{Proliferation and differentiation of rat bone MSCs (rBMSCs) on KGN-PCL membranes}

The effects of KGN-PCL scaffolds on the proliferation of rBMSCs were investigated and are shown in Fig. 1C. The rBMSCs proliferated on all aligned PCL electrospun membranes, which increased with time from day 1 to 7 . In addition, different amounts of KGN had similar effects on the proliferation of rBMSCs.

In addition, the effects of all aligned PCL electrospun membranes containing different amount of KGN $(0-100 \mu \mathrm{M})$ on chondrogenic and tenogenic differentiation of rBMSCs are shown in Fig. 2, including the expression of three specific chondrocyte-related genes, Aggrecan (Fig. 2A), COL2 (Fig. 2B), SRY-box 9 (SOX9, Fig. 2C), and tenocyte-related gene scleraxis
bHLH transcription factor (SCX, Fig. 2D). The results suggested that KGN-PCL containing $10 \mu \mathrm{M}$ KGN showed no stimulatory effects on the all three chondrocyte-related genes compared to the pure PCL membrane. When the concentration of KGN was increased to $50 \mu \mathrm{M}$, although the KGN-PCL membranes still did not upregulate the gene expression of Aggrecan and SOX9 from rBMSCs, they upregulated the gene expression of COL2 compared to that of the pure PCL membranes. Further increase in the KGN concentration of the KGN-PCL membranes to 100 $\mu \mathrm{M}$, significantly stimulated the expression of all three chondrocyte-related genes in rBMSCs compared with that of the pure PCL membranes. This finding indicated that $100 \mu \mathrm{M} \mathrm{KGN}$ was the optimal concentration to promote chondrogenisis of rBMSCs. Higher doses of KGN decreased the biomechanical 
properties of the structures according to a previous research study. ${ }^{13}$ The effects of PCL and KGN-PCL membranes on the expression of the tenocyte-related gene SCX is illustrated in Fig. 2D. The KGN-PCL using electrospun fibrous membrane did not improve the tenogenic effects of rBMSCs as there was no significant difference among PCL membranes containing different amount of KGN (0-100 $\mu \mathrm{M})$ (Table 1).

\section{Histomorphometric and immunohistochemistry analysis}

Hematoxylin and eosin (H\&E)-stained images are shown in Fig. 3. After the electrospun membranes were implanted for 2 weeks, few chondrocytes were observed in all groups. Compared with the control and pure PCL group, the tendon (indicated by " $T$ " in the images)-bone (indicated by "B" in the images) interface (indicated by "I" in the images) in the KGN-PCL group was filled with fibroblasts and blood vessels, as shown in the images. The membranes were still clearly observed in the RCT site, as indicated by arrow heads in the images. After 4 weeks, bone grew into the interface area and the dividing line between tendon and bone became unclear in all groups. In the KGN-PCL group, more fibrocartilage zones and oriented cells were observed than in the control and pure PCL groups. After 8 weeks, the membranes had degraded in all groups and were not observed in the samples. In the KGN-PCL group, the fibrocartilage zone became more similar to that in the normal tendon-bone interface and the cells aligned more regularly than those in the control and pure PCL groups.

New fibrocartilage formation was determined using safranin $\mathrm{O} /$ fast green staining (Fig. 4A) and the expression of glycosaminoglycan (red stained area) at the tendon-bone healing interface at all time points was quantitatively determined based on the glycosaminoglycan-positive stained surface area (Fig. 4B). After 2 weeks, the glycosaminoglycan-positive stained surface in the KGN, PCL, and control groups was $361694 \pm$ $26244,279195 \pm 28105$, and $196728 \pm 24193 \mu \mathrm{m}^{2}$, respectively. After 4 weeks, the glycosaminoglycan-positive stained area surface in KGN group, PCL group and control group was $528527 \pm 25427,456748 \pm 22593$, and $349650 \pm 26940 \mu \mathrm{m}^{2}$, respectively. At 8 weeks the glycosaminoglycan positive staining area surface in KGN group, PCL group and control group was $643125 \pm 49970 \mu \mathrm{m}^{2}, 556266 \pm 36754 \mu \mathrm{m}^{2}$, and $470534 \pm$ $36161 \mu \mathrm{m}^{2}$, respectively. All groups showed increased expression of glycosaminoglycan with time, and that of the KGN-PCL group was consistently highest, which indicated that the KGNPCL membrane improved cartilage regeneration. Furthermore, the expression of glycosaminoglycan in the PCL group was higher than that in control group was at all time points, which suggested that aligned PCL membranes also stimulated cartilage regeneration.
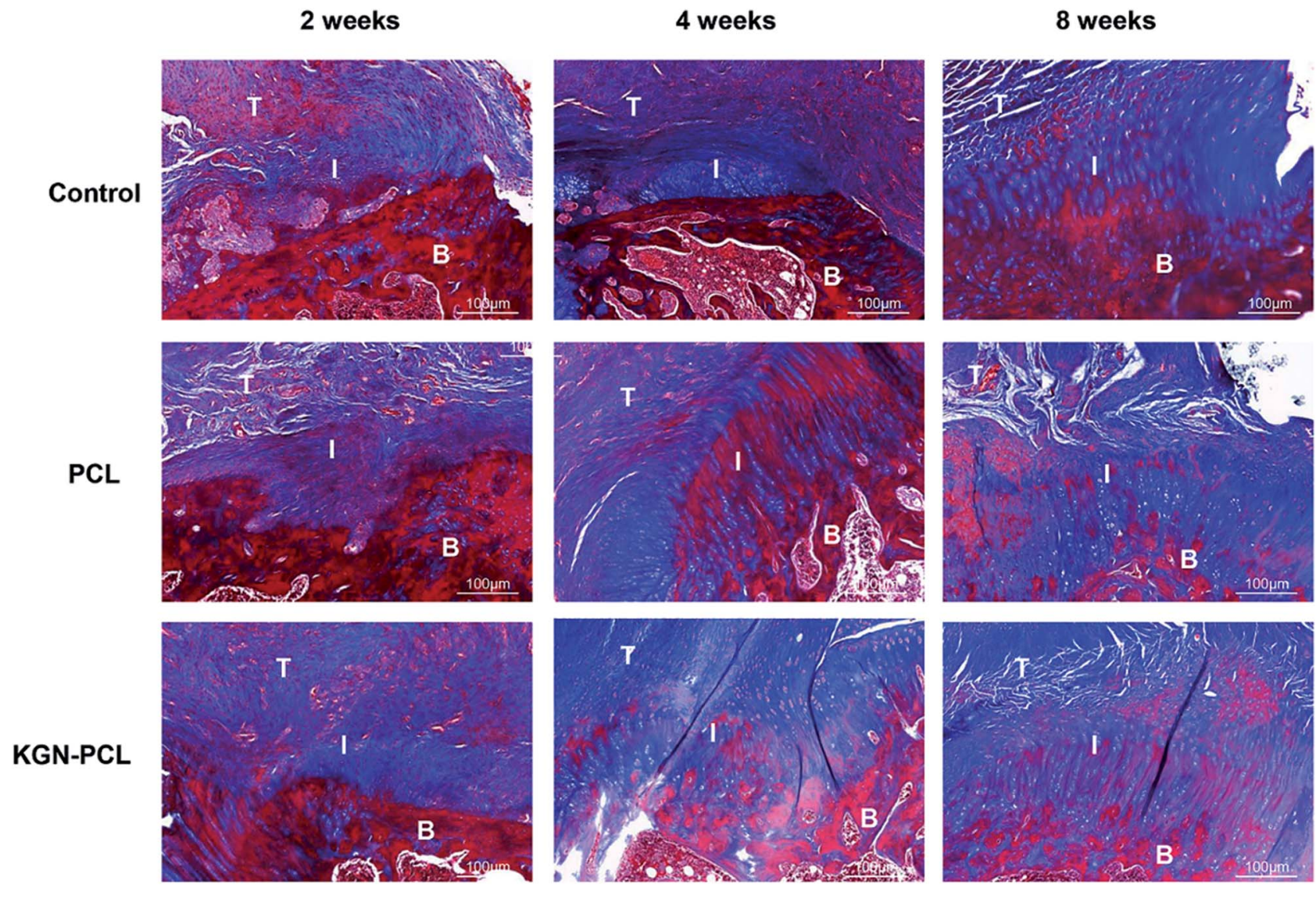

Fig. 5 Histology of rotator cuff tendon-bone healing enthesis, Masson-trichrome stained at 2, 4, and 8 weeks after the surgery. T: tendon; I: interface; B: bone. 
Novel collagen-fibril formation was further determined using Masson trichrome staining and the results are shown in Fig. 5. After 2 weeks, the KGN-PCL group showed the highest level of collagen-fibril among all the groups although the levels were not high in all groups. After 4 and 8 weeks, the amount of collagen-fibril in all groups increased with time. Compared with the control and PCL groups, there were more collagen fibers in the tendon-bone interface of the KGN-PCL group. In addition, the collagen-fibrils in the KGN-PCL and PCL groups were much better organized than those in the control groups.

The immunohistochemical staining demonstrated the COL1 protein expression of tenocytes and fibroblasts. Fig. 6 shows that there was more COL1 in the tendon tissues than there was in the TBJs in all groups at all time points. At 2 weeks, there was no significant difference in COL1 expression in the different groups. At 4 and 8 weeks, the expression of COL1 increased with time in all groups at the TBJ. In addition, the expression of COL1 in the KGN-PCL and PCL groups was higher than that in the control group was. COL2 immunohistochemical staining demonstrated the COL2 protein expression in chondrocytes. It can be seen in Fig. 7 that the new fibrocartilage formation increased with time at the TBJ in all groups. In addition, there was a higher expression of COL2 in the TBJs of the KGN-PCL than in other groups at all time points. This observation indicated that the KGN-PCL membranes stimulated fibrocartilage more in the KGN-PCL group than in the PCL and control groups of formation.

\section{Biomechanical testing}

As shown in Fig. 8A, the ultimate load to failure in KGN-PCL group showed no significant difference compared to that in the control and PCL group at 2 weeks after the surgery. At 4 weeks, the ultimate load to failure in the KGN, PCL, and control group was $22.1 \pm 0.5 \mathrm{~N}, 19.4 \pm 1.4 \mathrm{~N}$ and $15.3 \pm 0.7 \mathrm{~N}$, respectively. At 8 weeks, the ultimate load to failure in the KGN, PCL, and control groups were $29.7 \pm 1.6,25.5 \pm 0.2,2$ and $0.7 \pm$ $0.5 \mathrm{~N}$, respectively. It can be seen that the ultimate load to failure increased with time in all groups. After 4 and 8 weeks, the ultimate load to failure in PCL group was higher than that in the control group, which indicated the aligned PCL fibrous membrane could improve the biomechanical properties in repairing site. Furthermore, the ultimate load to failure in the PCL-KGN group was highest among the three groups at 4 and 8 weeks, which suggested that the aligned KGN-PCL group could further improve the biomechanical properties, leading to effective repair of RCT.

The result of the examination of the cross-sectional area of the tendon-bone healing enthesis is showed in Fig. 8B. At 2 weeks, there was no difference between the control and experimental groups. At 4 weeks, the PCL and KGN-PCL groups both

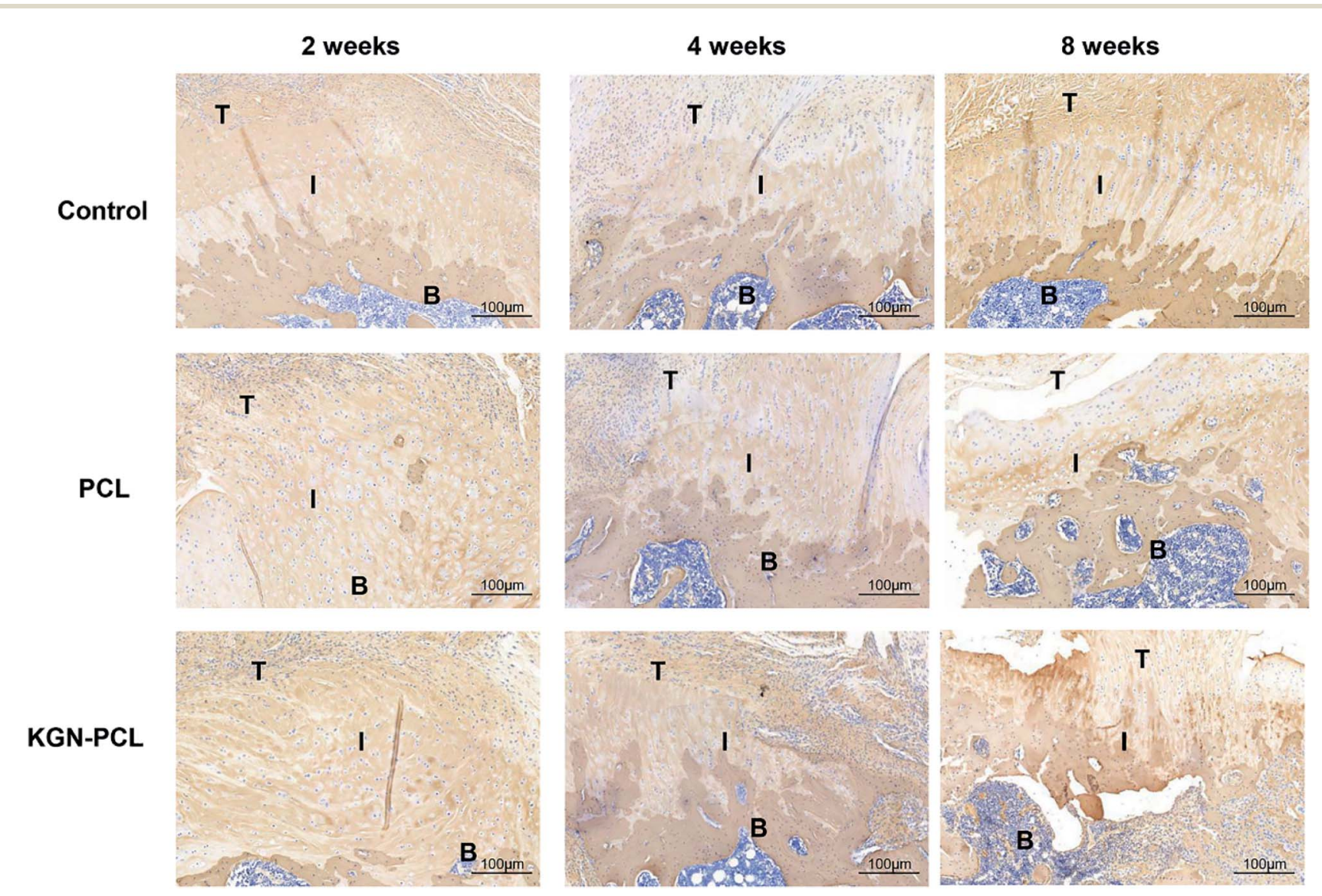

Fig. 6 Immunohistochemistry assay of collagen I in rotator cuff tendon-bone enthesis at 2, 4, and 8 weeks after the surgery. T: Tendon; I: interface; B: bone. 
showed significant difference compared to the control group while there was no difference between the PCL and KGN-PCL groups. At 8 weeks, the cross-sectional area of KGN-PCL group was significantly higher than that of the other two groups, which indicated that KGN-PCL influenced tissue regeneration 8 weeks after the operation.

\section{Discussion}

To date, RCT is still a major challenging disease in clinics as the rotator cuff can easily rupture after surgery. ${ }^{39,40}$ The TBJs are hard to heal at the repair site because the fibrocartilage transition zone in TBJs cannot completely regenerate when the tendon-bone healing is according to the results of the histopathological studies., ${ }^{\mathbf{8} 9}$ Different methods have proposed improve tendon-bone healing, including application of cells, biomaterials, and growth factors. ${ }^{41-44}$ It has been reported that aligned electrospun fibrous membrane can enhance cell proliferation and promote tenogenesis due to its instructive microenvironment, ${ }^{29,30}$ leading to the repair of RCT. However, single application of aligned electrospun fibrous membrane to tendon-bone healing site is insufficient for stimulating the regeneration of fibrocartilage transition zone. As electrospun fibrous membranes have been widely used as a sustained drug delivery system ${ }^{19}$ and $\mathrm{KGN}$ has been reported to be able to induce chondrogenic differentiation of BMSCs and stimulate formation of cartilage tissues. ${ }^{13}$ Combined use of aligned electrospun fibrous membrane and KGN in tendon-bone healing was proposed in the present study. Our findings first demonstrated that KGN could constantly release from aligned fibers in 20 days after it was encapsulated in aligned electrospun fibrous membranes. In addition, the aligned electrospun fibrous scaffolds loading KGN promoted the proliferation of rBMSCs and induce the chondrogenic and tenogenic differentiation of rBMSCs. After implantation, compared to the PCL membranes, KGN-PCL membranes increased the amount of collagen fibers and promoted healing of the fibrocartilage zone, resulting in the improvement of pull-out strength at healing enthesis of TBJs.

KGN has been proven effective in tendon-bone healing; however, single topical injections into the repair site of RCT may not be optimal. ${ }^{14}$ This is because KGN has not been confirmed to act at the repair site ${ }^{15}$ because of the circulation of body fluids. To address solve this problem, growth factors or biological small molecules were encapsulated in electrospun fibrous membranes to determine the local release of the biological molecules and finally improve tendon-bone regeneration. ${ }^{45}$ For example, basic fibroblast growth factors have been loaded into electrospun fibrous membrane to enhance tendonbone healing in RCT. ${ }^{46}$ In this study, KGN was encapsulated in aligned electrospun fibrous membrane, which prevented it from diffusing into the surrounding area and maintained its
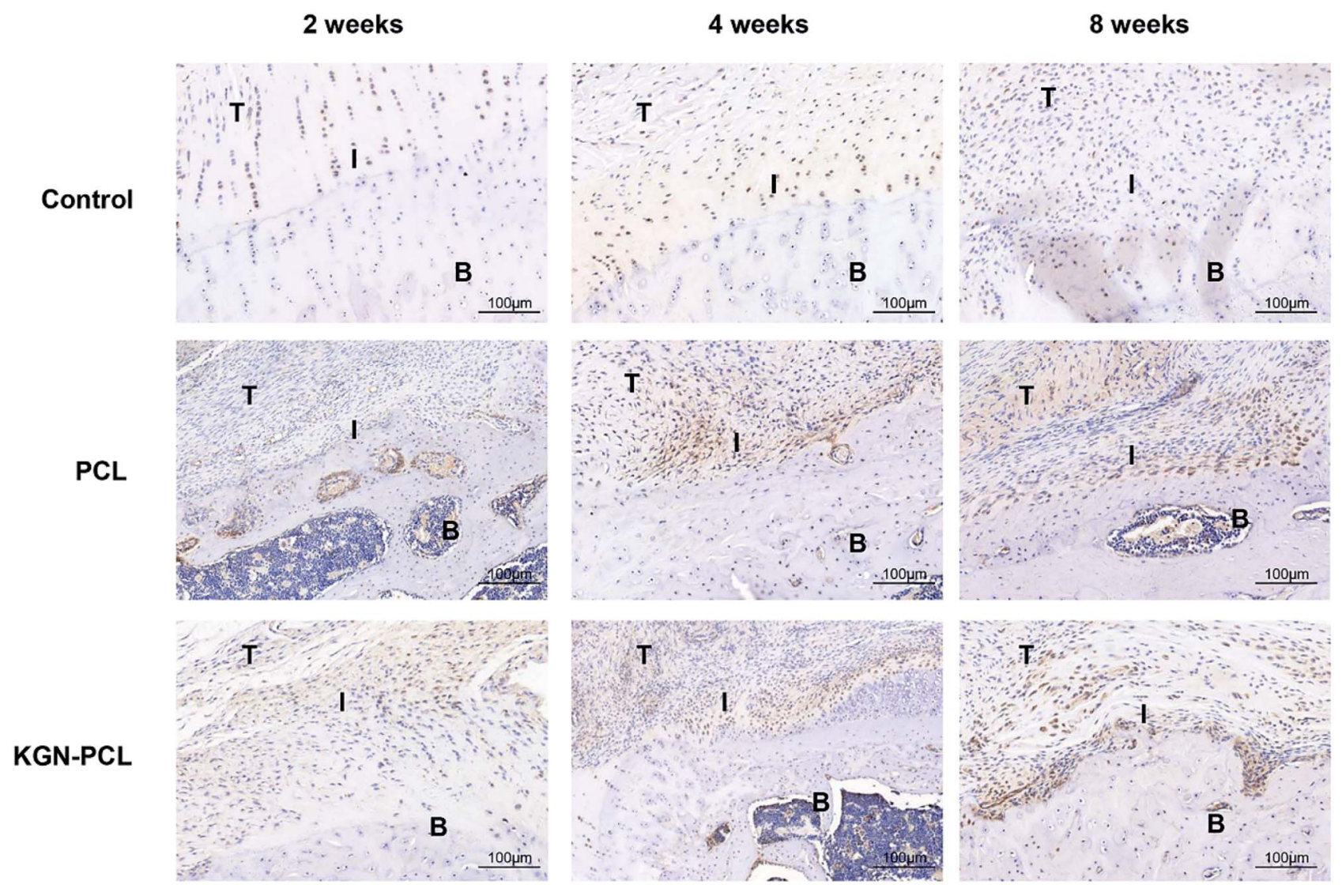

Fig. 7 Immunohistochemistry assay of collagen II in rotator cuff tendon-bone enthesis at 2, 4, and 8 weeks after the surgery T: tendon; I: interface; B: bone. 

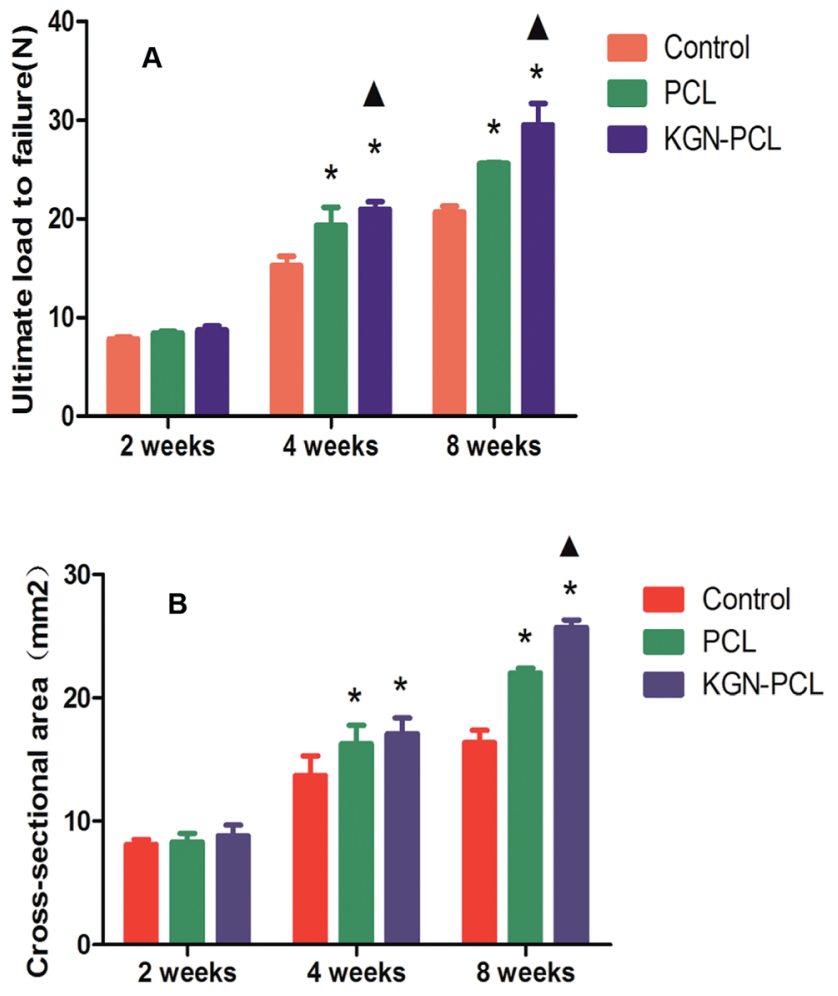

Fig. 8 Result of biomechanical test of tendon-bone junction. (A) Ultimate load to failure. (B) Cross-sectional area. The results are the means \pm standard deviation (*: $P<0.05$ versus control group; $\boldsymbol{\Delta}: P<$ 0.05 versus $\mathrm{PCL}$ group. $n=5$ each group).

biological effects at the repair site for 20 days. Therefore, aligned electrospun fibrous membranes prolonged the KGN duration of action, which might be one of the reasons for the enhanced cartilage formation in tendon-bone enthesis.

The failure rate of tendon-bone healing after RCT repair surgeries is still high because the fibrocartilage transition zone in tendon-bone enthesis is hard to regenerate. ${ }^{2-5,47}$ The fibrocartilage transition zone can enhance the strength of tissue bonding between tendon and bone. In this study, local application of aligned PCL electrospun fibrous membranes improved fibrocartilage formation and collagen organization at the tendon-bone enthesis with the control group. We supposed that the aligned PCL electrospun fibrous membrane served as a provisional matrix in the phase of tendon-bone healing and promoted tenogenesis, leading to the alignment of newly synthesized collagen fibers. Since PCL electrospun fibrous membrane with aligned nanofibers have the structural stimulatory effects and KGN has the chemical stimulatory effects on RCT repairs KGN was previously shown to promote stem cells proliferation and differentiation into chondrocytes. ${ }^{13,14,17} \mathrm{We}$ found that the effects of KGN on chondrogenic differentiation of rBMSCs was dose-dependent and high-dose KGN $(100 \mu \mathrm{M})$ showed the highest stimulatory effects, which may have enhanced the enthesis healing by improving fibrocartilage formation and collagen organization.

Biomechanical testing is used to evaluate whether the biomechanical properties of the repair site can be improved by the application of scaffolds. Biomechanical properties are important for tendon-bone healing as they can confirm that the tendon-bone enthesis are not lax and forces are efficiently transmitted across the enthesis to the bone. ${ }^{48} \mathrm{Xu}$ et al. reported that aligned poly(L-lactide-co-\&-caprolactone)/collagen scaffolds improved the biomechanical properties of rabbit patella tendons. ${ }^{49}$ In the present study, the cross-sectional area and ultimate load to failure of RCT repaired with pure PCL were higher than those of the control 4 and 8 weeks, which indicated that the electrospun membranes with aligned nanofibers enhanced the mechanical strength of regenerated RCs. Interesting, the cross-sectional area and ultimate load to failure of RCT repaired in the KGN-PCL groups were even higher than those of PCL group were. This finding indicates that the combined use of KGN and electrospun membranes with aligned nanofibers could significantly improve the biomechanical properties, leading the effective repair of RCT.

\section{Conclusion}

In this study, we first combined a PCL electrospun fibrous membrane with aligned nanofibers and KGN for healing tendon-bone interface in RCT. In this system, the PCL electrospun fibrous membrane with aligned nanofibers not only acted as the KGN delivery system to ensure its constant release in the defect site for 20 days but also might have served as a structural guide for the alignment of newly synthesized collagen fibers in the tendon-bone interface. In addition, KGN had chemical stimulatory effects on fibrocartilage synthesis and $100 \mu \mathrm{M}$ stimulate the chondrogenic differentiation of rBMSCs. Therefore, PCL electrospun fibrous membrane with aligned nanofibers encapsulating KGN could enhance tendon-bone healing in RCT by promoting fibrocartilage formation and collagen organization and strengthen the healing enthesis.

\section{Conflicts of interest}

The authors declare no conflict of interest.

\section{Acknowledgements}

The work was supported by the National Key Research and Development Program of China (No: 2016YFC1100201), the National Natural Science Foundation of China (81572106, 31470918, and 31171024) and Program of Shanghai Academic/ Technology Research Leader.

\section{References}

1 B. G. Domb, R. E. Glousman, A. Brooks, M. Hansen, T. Q. Lee and N. S. ElAttrache, J. Bone Jt. Surg., 2008, 90, 35-39.

2 C. Gerber, B. Fuchs and J. Hodler, J. Bone Jt. Surg., 2000, 82, 505-515.

3 P. Boileau, N. Brassart, D. J. Watkinson, M. Carles, A. M. Hatzidakis and S. G. Krishnan, J. Bone Jt. Surg., 2005, 87, 1229-1240.

4 K. Accousti and E. Flatow, Instr. Course Lect., 2007, 56, 3-12. 
5 R. H. Cofield, J. Parvizi, P. J. Hoffmeyer, W. L. Lanzer, D. M. Ilstrup and C. M. Rowland, J. Bone Jt. Surg., 2001, 83, 71-77.

6 J. H. Oh, S. H. Kim, H. M. Ji, K. H. Jo, S. W. Bin and H. S. Gong, Arthroscopy, 2009, 25, 30-39.

7 N. Hibino, Y. Hamada, K. Sairyo, K. Yukata, T. Sano and N. Yasui, J. Bone Jt. Surg., Br. Vol., 2007, 89, 1539-1544.

8 K. S. Leung, L. Qin, L. K. Fu and C. W. Chan, Clin. Biomech., 2002, 17, 594-602.

9 M. W. N. Wong, L. Qin, K. M. Lee, K. O. Tai, W. S. Chong, K. S. Leung and K. M. Chan, Clin. Orthop. Relat. Res., 2003, 413, 291-302.

10 L. M. Galatz, L. J. Sandell, S. Y. Rothermich, R. Das, A. Mastny, N. Havlioglu, M. J. Silva and S. Thomopoulos, J. Orthop. Res., 2006, 24, 541-550.

11 K. Atesok, F. H. Fu, M. R. Wolf, M. Ochi, L. M. Jazrawi, M. N. Doral, J. H. Lubowitz and S. A. Rodeo, J. Bone Jt. Surg., 2014, 96, 513-521.

12 H. J. Seeherman, J. M. Archambault, S. A. Rodeo, A. S. Turner, L. Zekas, D. D'augusta, X. J. Li, E. Smith and J. M. Wozney, J. Bone Jt. Surg., 2008, 90, 2206-2219.

13 K. Johnson, S. Zhu, M. S. Tremblay, J. N. Payette, J. Wang, L. C. Bouchez, S. Meeusen, A. Althage, C. Y. Cho and $\mathrm{X}$. Wu, Science, 2012, 336, 717-721.

14 J. Zhang and J. H. Wang, Bone Res., 2014, 2, 14008.

15 G. Mohan, S. Magnitsky, G. Melkus, K. Subburaj, G. Kazakia, A. J. Burghardt, A. Dang, N. E. Lane and S. Majumdar, J. Orthop. Res., 2016, 34, 1780-1789.

16 Y. Zhou, J. Zhang, J. Yang, M. Narava, G. Zhao, T. Yuan, H. Wu, N. Zheng, M. V. Hogan and J. H. C. Wang, J. Tissue Eng. Regener. Med., 2017, 11, 3445-3456.

17 J. Zhang, T. Yuan, N. Zheng, Y. Zhou, M. Hogan and J. H. Wang, Bone Jt. Res., 2017, 6, 231-244.

18 X. Y. Zhang, S. Q. Wang, L. X. Xu, L. Feng, Y. Ji, L. Tao, S. X. Li and Y. Wei, Nanoscale, 2012, 4, 5581-5584.

19 Y. L. Liu, K. L. Ai and L. H. Lu, Chem. Rev., 2014, 114, 50575115.

20 M. Liu, G. Zeng, K. Wang, Q. Wan, L. Tao, X. Zhang and Y. Wei, Nanoscale, 2016, 8, 16819-16840.

21 M. Liu, J. Ji, X. Zhang, X. Zhang, B. Yang, F. Deng, Z. Li, K. Wang, Y. Yang and Y. Wei, J. Mater. Chem. B, 2015, 3, 3476-3482.

22 Y. Shi, M. Liu, F. Deng, G. Zeng, Q. Wan, X. Zhang and Y. Wei, J. Mater. Chem. B, 2017, 5, 194-206.

23 L. Huang, M. Liu, H. Huang, Y. Wen, X. Zhang and Y. Wei, Biomacromolecules, 2018, 19, 1858-1868.

24 Q. Huang, M. Liu, J. Chen, Q. Wan, J. Tian, L. Huang, R. Jiang, Y. Wen, X. Zhang and Y. Wei, Appl. Surf. Sci., 2017, 419, 35-44.

25 F. Zamani, M. Amani-Tehran, M. Latifi and M. A. Shokrgozar, J. Mater. Sci.: Mater. Med., 2013, 24, 1551-1560.

26 C. Hu and W. Cui, Adv. Healthcare Mater., 2012, 1, 809-814.

27 S. B. Orr, A. Chainani, K. J. Hippensteel, A. Kishan, C. Gilchrist, N. W. Garrigues, D. S. Ruch, F. Guilak and D. Little, Acta Biomater., 2015, 24, 117-126.
28 S. Shang, F. Yang, X. Cheng, X. F. Walboomers and J. A. Jansen, Eur. Cells Mater., 2010, 19, 180-192.

29 K. L. Moffat, A. S.-P. Kwei, J. P. Spalazzi, S. B. Doty, W. N. Levine and H. H. Lu, Tissue Eng., Part A, 2008, 15, 115-126.

30 Z. Yin, X. Chen, J. L. Chen, W. L. Shen, T. M. H. Nguyen, L. Gao and H. W. Ouyang, Biomaterials, 2010, 31, 2163-2175.

31 A. Luciani, V. Coccoli, S. Orsi, L. Ambrosio and P. A. Netti, Biomaterials, 2008, 29, 4800-4807.

32 S. Martinez-Diaz, N. Garcia-Giralt, M. Lebourg, J.-A. GomezTejedor, G. Vila, E. Caceres, P. Benito, M. Monleon Pradas, X. Nogues and J. L. Gomez Ribelles, Am. J. Sports Med., 2010, 38, 509-519.

33 C. X. Lam, D. W. Hutmacher, J. T. Schantz, M. A. Woodruff and S. H. Teoh, J. Biomed. Mater. Res., Part A, 2009, 90, 906-919.

34 M. Ni, Y. Rui, Q. Chen, Y. Wang and G. Li, Zhongguo Xiufu Chongjian Waike Zazhi, 2011, 25, 1103-1109.

35 Y.-F. Rui, P. P. Y. Lui, G. Li, S. C. Fu, Y. W. Lee and K. M. Chan, Tissue Eng., Part A, 2010, 16, 1549-1558.

36 J. Carpenter, S. Thomopoulos, C. Flanagan, C. DeBano and L. Soslowsky, J. Shoulder Elb. Surg., 1998, 7, 599-605.

37 L. J. Soslowsky, J. E. Carpenter, C. M. DeBano, I. Banerji and M. R. Moalli, J. Shoulder Elb. Surg., 1996, 5, 383-392.

38 C.-H. Chen, C.-H. Chang, K.-C. Wang, C.-I. Su, H.-T. Liu, C.-M. Yu, C.-B. Wong, I.-C. Wang, S. W. Whu and H.-W. Liu, Knee Surg. Sport. Traumatol. Arthrosc., 2011, 19, 1597-1607.

39 L. M. Galatz, C. M. Ball, S. A. Teefey, W. D. Middleton and K. Yamaguchi, J. Bone Jt. Surg., 2004, 86, 219-224.

40 M. E. Angeline and S. A. Rodeo, Clin. Sports Med., 2012, 31, 645-663.

41 J. P. Spalazzi, E. Dagher, S. B. Doty, X. E. Guo, S. A. Rodeo and H. H. Lu, J. Biomed. Mater. Res., Part A, 2008, 86, 1-12.

42 R. Di Liddo, P. Paganin, S. Lora, D. Dalzoppo, C. Giraudo, D. Miotto, A. Tasso, S. Barbon, M. Artico and E. Bianchi, Int. J. Mol. Med., 2014, 34, 1537-1546.

43 X. Cheng, C. Tsao, V. L. Sylvia, D. Cornet, D. P. Nicolella, T. L. Bredbenner and R. J. Christy, Acta Biomater., 2014, 10, 1360-1369.

44 C. Cicione, E. Muiños-López, T. Hermida-Gómez, I. FuentesBoquete, S. Díaz-Prado and F. J. Blanco, Cell Tissue Banking, 2015, 16, 195-207.

45 L. Smith, Y. Xia, L. M. Galatz, G. M. Genin and S. Thomopoulos, Connect. Tissue Res., 2012, 53, 95-105.

46 S. Zhao, J. Zhao, S. Dong, X. Huangfu, B. Li, H. Yang, J. Zhao and W. Cui, Int. J. Nanomed., 2014, 9, 2373.

47 S. Thomopoulos, G. M. Genin and L. M. Galatz, J. Musculoskeletal Neuronal Interact., 2010, 10, 35.

48 S. Sahoo, S. L. Toh and J. C. Goh, Biomaterials, 2010, 31, 2990-2998.

49 Y. Xu, S. Dong, Q. Zhou, X. Mo, L. Song, T. Hou, J. Wu, S. Li, Y. Li and P. Li, Biomaterials, 2014, 35, 2760-2772. 